Sir,

\section{Determination of seasonal allergic conjunctivitis variation using internet search engine data}

A common and potentially debilitating disease, seasonal allergic rhinoconjunctivitis (SAR) can greatly affect the quality of life and is associated with lost productivity. ${ }^{1}$ Many sufferers are at the milder end of the disease spectrum and 'fly under the radar' of health services. Consequently, the epidemiology of seasonal allergic conjunctivitis has been difficult to study without expensive large-scale health surveys. ${ }^{2}$ Knowledge of the likely timing of local SAR activity peaks is useful at a number of levels. It can help in workforce planning and training. It also allows optimal timing of the use of anti-allergy treatments such as topical mast cell stabilisers, which may take several weeks to have maximal effect. Here we use a validated method of identifying periods of high disease activity to describe the seasonal pattern of SAR in the United Kingdom.

Analysis of internet search engine activity has been used to identify outbreaks of seasonal influenza. ${ }^{3}$ We searched the Google trends application (Google, Mountain View, CA, USA) for the terms 'hay fever' and 'hayfever', limited to the United Kingdom and the months of January 2008 through December 2010 (http:/ / www.google.com/trends (accessed 1 February 2011)). The combined results demonstrate a consistent pattern of online search engine activity (Figure 1). No search activity is seen during Winter. Searches begin in Spring, with a small peak in the search volume index in late April/early May and a larger peak in mid-June. The smaller peak occurs during the peak tree pollen season (eg, birch), while the larger peak occurs during the peak grass pollen season.

We acknowledge that this method is limited by the facts that the study population excludes those without internet access and that the data are from a single search engine, introducing a degree of bias. Further inaccuracy may, in theory, arise from any significant delay between symptom onset and internet search by the sufferer. However, we have previously demonstrated that such

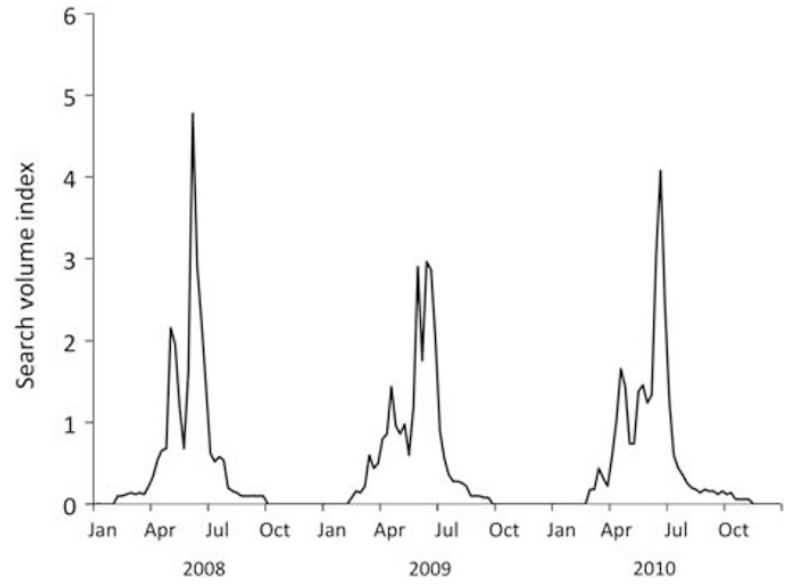

Figure 1 Online search-engine-combined activity for 'hay fever' and 'hayfever' from 2008 to 2010 in the United Kingdom, using the Google search engine. The scale on the $y$ axis compares the activity with that at a fixed point in time. analysis is as effective as a large-scale cross-sectional epidemiological study in identifying the peak seasonal activity of $\mathrm{SAR}^{4}$ and feel that this inadvertent mass collaboration provides useful information as to the timing of peak symptoms of allergic rhinoconjunctivitis in the United Kingdom.

\section{Conflict of interest}

The authors declare no conflict of interest.

\section{References}

1 Fireman P. Treatment of allergic rhinitis: effect on occupation productivity and work force costs. Allergy Asthma Proc 1997; 18(2): 63-67.

2 Singh K, Axelrod S, Bielory L. The epidemiology of ocular and nasal allergy in the United States, 1988-1994. J Allergy Clin Immunol 2010; 126(4): 778-783 e6.

3 Ginsberg J, Mohebbi MH, Patel RS, Brammer L, Smolinski MS, Brilliant L. Detecting influenza epidemics using search engine query data. Nature 2009; 457(7232): 1012-1014.

4 Flynn TH. Identifying seasonal variation in allergic rhinoconjunctivitis. J Allergy Clin Immunol 2011; 127(5): 1314-1315.

R Imonikhe ${ }^{1,2}$, TH Flynn ${ }^{1,2}$ and AA Mearza ${ }^{1,2}$

${ }^{1}$ The Western Eye Hospital, London, UK

${ }^{2}$ Department of Ophthalmology, Charing Cross

Hospital, London, UK

E-mail: ali.mearza@imperial.nhs.uk

Eye (2011) 25, 1663; doi:10.1038/eye.2011.249; published online 14 October 2011

\section{Sir Bevacizumab and type 1 idiopathic macular telangiectasia}

In reply to Takayama et $a l_{,}^{1}$ and to further support findings of Gamulescu et $a l^{2}{ }^{2}$ we wish to report a case of type 1 idiopathic macular telangiectasia (IMT) successfully treated with intravitreal bevazinumab.

\section{Case report}

A 76-year-old man with hypertension and ischaemic heart disease came with painless, progressive blurring of vision in the right eye for 2 months. His best-corrected visual acuity was $6 / 60$. There was macular oedema with underlying microaneurysms and hard exudates. Cystoid macular oedema was seen on optical coherence tomography (OCT). Fundus fluorescent angiography (FFA) showed multiple areas of telangiectasia temporal to the fovea with dye leakage at the mid- and late-phase angiograms (Figures $1 \mathrm{a}$ and $\mathrm{b}$ ).

The patient was diagnosed with chronic cystoid macular oedema secondary to type 1 IMT and was given one dose of intravitreal bevacizumab $(1.25 \mathrm{mg}$ per $0.05 \mathrm{ml}$ ) to his right eye. Visual acuity improved to $6 / 36$ at one month and a second dose of intravitreal bevacizumab was given. Vision further improved and 